\title{
Clinical and pathophysiologic relevance of autoantibodies in rheumatoid arthritis
}

\author{
Sara de Brito Rocha*, Danielle Cristiane Baldo and Luis Eduardo Coelho Andrade
}

\begin{abstract}
Rheumatoid arthritis (RA) is an autoimmune/inflammatory disease affecting 0.5 to $1 \%$ of adults worldwide and frequently leads to joint destruction and disability. Early diagnosis and early and effective therapy may prevent joint damage and lead to better long-term results. Therefore, reliable biomarkers and outcome measures are needed. Refinement of the understanding of molecular pathways involved in disease pathogenesis have been achieved by combining knowledge on RA-associated genes, environmental factors and the presence of serological elements. The presence of autoantibodies is a distinctive feature of RA. Rheumatoid Factor and Anti-Citrullinated Protein Antibodies are the two most remarkable autoantibodies in RA and provide different clinical and pathophysiological information. They precede the onset of disease symptoms and predict a more severe disease course, indicating a pathogenetic role in RA. Therefore, they promote a more accurate prognosis and contribute for a better disease management. Several RA-associated autoantibody systems have been identified: Anti-Carbamylated Antibodies, Anti-BRAF, Anti-Acetylated, Anti-PAD4 antibodies and others. Hopefully, the characterization of a comprehensive array of novel autoantibody systems in RA will provide unique pathogenic insights of relevance for the development of diagnostic and prognostic approaches compatible with an effective personalized medicine.
\end{abstract}

Keywords: Rheumatoid arthritis, Autoantibodies, ACPA, Anti-CarP, Rheumatoid factor, Personalized medicine

\section{Introduction}

Rheumatoid arthritis (RA) is an autoimmune/inflammatory disease affecting 0.5 to $1 \%$ of adults worldwide [1]. Women are three times more susceptible than men and the disease is more frequent at the age of $40-50$ years [1]. RA frequently leads to joint destruction and disability [1]. Early diagnosis and early and effective therapy may prevent joint damage and lead to better long-term results [1]. Optimal management of RA is needed within 3 to 6 months after onset of disease, since substantial irreversible joint damage has been shown to occur within the first 2 years. Therefore, reliable biomarkers and outcome measures are needed in order to establish early diagnosis, assess prognosis, and achieve a better disease management $[2,3]$.

The etiology of rheumatoid arthritis (RA) is not known, although genetic, environmental factors and serological elements have been identified to play a role in disease initiation and progression [4]. Smoking is now

\footnotetext{
* Correspondence: sb.rocha@gmail.com
Rheumatology Division, Escola Paulista de Medicina, Universidade Federal de

* Correspondence: sb.rocha@gmail.com
Rheumatology Division, Escola Paulista de Medicina, Universidade Federal de São Paulo, Disciplina de Reumatologia, Rua Botucatu 740, 30 andar, São Paulo, SP ZIP:04023-062, Brazil
}

(c) The Author(s). 2019 Open Access This article is distributed under the terms of the Creative Commons Attribution 4.0 International License (http://creativecommons.org/licenses/by/4.0/), which permits unrestricted use, distribution, and reproduction in any medium, provided you give appropriate credit to the original author(s) and the source, provide a link to the Creative Commons license, and indicate if changes were made. The Creative Commons Public Domain Dedication waiver (http://creativecommons.org/publicdomain/zero/1.0/) applies to the data made available in this article, unless otherwise stated.

a well-known environmental trigger [5]. Genetic contribution is estimated around 50 to $60 \%$ and therefore, genetic factors have an important impact on susceptibility to RA [4]. The strongest predisposing gene variants are found in the human leukocyte antigen (HLA) genes, accounting for 30 to $50 \%$ of overall genetic susceptibility to RA [6]. Multiple RA risk alleles within the HLA-DRB1 gene share a conserved amino acid sequence, leading to the "shared epitope" (SE) concept [6]. The presence of one HLA SE allele confers an odds ratio to develop RA around 4, and the presence of two SE copies increases the odds ratio to approximately 11 [7].

The presence of autoantibodies is a distinctive feature of RA. The two autoantibody systems most commonly used as an aid for diagnosing/classifying RA are rheumatoid factor (RF) and anti-citrullinated protein antibodies (ACPA). They precede the onset of disease symptoms and predict a more severe disease course, indicating a pathogenic role in RA. Therefore, they promote a more accurate prognosis and contribute for a better disease management. Their importance was recently emphasized by the inclusion of ACPA alongside the previously included RF on ACR/EULAR 2010 RA diagnostic criteria [8]. 
The hypothesis that autoantibodies may play a pathophysiologic role has been fueled by the discovery of strong associations linking the HLA-DRB1 SE and PTPN22 alleles, smoking and the presence of autoantibodies, in particular ACPA [7]. Recently, other forms of post-translational modification have been associated with the generation of RA-relevant autoantigens and autoantibodies that can be used as useful biomarkers [9]. In predisposed subjects, failure in keeping self-tolerance might be elicited by post-translational modifications, since these processes might promote generation of neoepitopes and neo-(auto)antigens [9].

This article provides an update on the state of the art on autoantibodies in rheumatoid arthritis (RA).

\section{Rheumatoid factor \\ Characteristics}

The first autoantibody discovered in RA patients is the RF. It was first described in 1940 as an antibody directed against serum gamma-globulins and promoted the agglutination of sheep red blood cells sensitized by subagglutinating doses of rabbit antibodies [10]. In 1948, these antibodies were described in patients with RA, and in 1952 they were called RF due to their strong association with RA [10]. RF are autoantibodies that directly bind to the Fc portion of the aggregated IgG and are locally produced by B cells present in lymphoid follicles and germinal center-like structures that develop in inflamed RA synovium $[10,11]$.

RF testing in RA patients has a sensitivity ranging from 60 to $90 \%$ and a specificity ranging from 48 to $92 \%$, according to different studies [10]. RF has limited specificity, since it can also be found in healthy controls and patients with other autoimmune diseases such as systemic lupus erythematosus and systemic sclerosis, and in non-autoimmune diseases, such as chronic infections and cancer $[4,10,12] \mathrm{RF}$ is found in multiple immunoglobulin isotypes (IgM, IgG and $\operatorname{IgA}$ ) wherein IgM-RF is the one usually measured in most clinical laboratories, being detected in $60-80 \%$ of RA patients [12]. The simultaneous occurrence of IgM, IgA, and IgG RF is present in up to $52 \%$ of RA patients but in fewer than $5 \%$ of patients with other connective tissue diseases. The combined occurrence of IgM and IgA RF has high diagnostic specificity for rheumatoid arthritis, but the presence of IgA and IgG RF isotypes in absence of IgM-RF is less specific, since they are also prevalent in patients with diverse connective tissue diseases [12, 13]. IgM-RF specificity increases considerably at high titres $[4,10,12]$. RF reactivity presents several differences in healthy and RA patients. Healthy subjects usually present poly-reactive, low affinity, low titer IgM class RF, whereas RA patients usually present more than one isotype RF at higher titer and with higher avidity [10]. It has been shown that high titer RF in healthy subjects is associated with increased risk of developing RA [14]. Finally, IgM RF has increased frequency in healthy elderly people, which suggests that they may be also related to the age-related immune deregulation [12, 14].

\section{Role in pathogenesis}

There is evidence supporting the concept that RF is a pathogenic autoantibody with a key role in the physiopathology of RA [15]. In normal conditions, transient production of low-affinity IgM RF is regularly induced by immune complexes [15] and polyclonal B-cell activators, such as bacterial lipopolysaccharides and Epstein-Barr virus [10]. The physiological role of RF under normal conditions includes promoting stability of IgG bound to solid surfaces, such as bacterial walls; enhancing immune complex clearance by increasing its stability and size; helping B cells uptake immune complexes, and thereby, efficiently present antigens to $\mathrm{T}$ cells; and facilitating complement fixation by binding to IgG containing immune complexes $[15,16]$. High affinity and high-titer RF in RA synovial fluid are believed to exert such functions in a pathogenic manner and thus to potentiate inflammation and antigen trapping in the joints [16]. In RA, RF may induce the formation of immune complexes at the sites of synovial inflammation, ensuing the activation of complement and leukocyte infiltration (Fig. 1) [16]. B cells with RF specificity migrate into the synovium of RA patients, presenting a variety of antigens to $\mathrm{T}$ cells and this may contribute to the perpetuation of local inflammatory responses and amplification of RF production in the synovium. Thus, RF may prolong B cell survival and hence maintain its own production $[11,15,16]$.

\section{Clinical relevance}

RF plays a pivotal role in the differential diagnosis and determination of prognosis of patients with arthritis [16]. It has been shown that RF is useful in predicting the development of RA, as the detection of IgM, IgA, and IgG RF

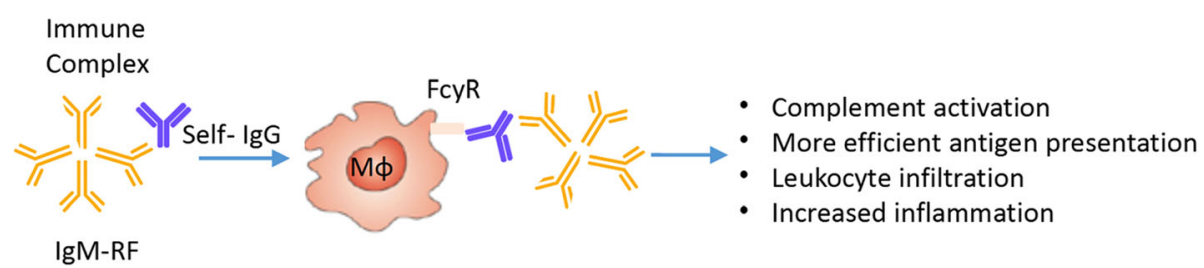

Fig. 1 The pathogenetic role of the rheumatoid factor: formation of immune complexes at the sites of synovial inflammation, activation of complement, and leukocyte infiltration, increasing inflammation 
may predate disease onset by years [10]. The pre-clinical appearance of RF isotypes in serum follows a specific sequential evolution: first IgM RF, then IgA RF, and finally IgG RF [17]. High titers of RF have been associated with worse prognosis, more aggressive articular disease, increased disease activity, reduced rates of remission, higher prevalence of extra-articular manifestations, and increased morbidity and mortality, especially when in combination with ACPA [11, 17, 18].

Some studies have shown that immunosuppressive treatment can decrease RF serum levels, but the clinical usefulness of RF in monitoring disease activity and treatment response is limited [18]. Progressive decrease in RF levels parallels the decrease of disease activity in patients treated with conventional disease modifying anti-rheumatic drugs (DMARDs) or biologic agents such as infliximab, etanercept and adalimumab $[4,10]$. The published data regarding the potential role of RF in predicting responses to antitumor necrosis factor alpha (TNF- $\alpha$ ) in controversial, as one study suggests that the presence of RF predicts a negative response [19], whereas two other studies show that RF positivity before therapy is insufficient to predict the therapeutic response [20, 21]. It has been reported that high IgA RF pre-treatment levels are associated with a poor clinical response to TNF- $\alpha$ inhibitors [10]. Since high serum levels of RF are predictors of more severe disease forms, it is expected that $\mathrm{B}$ cell-depleting therapy can have a beneficial effect. Indeed, RF positivity seems to predict better response to rituximab $[10,15,16]$ and to tocilizumab but not to abatacept $[10,22]$.

\section{ACPA}

\section{Characteristics}

The characterization of autoantibodies reacting with citrullinated peptides (ACPA) in RA was first reported in 1998 [9]. However, the history of ACPA starts in 1964 when fluorescence of anti-perinuclear factor (APF) was described in RA sera. Subsequently, anti-keratin antibodies (AKA) that had, as APF, a high specificity for RA, were reported. Over the years, other candidate citrullinated autoantigens have been identified, such as fibrinogen, vimentin, fibronectin and $\alpha$-enolase [23]. The Sa antigen/autoantibody system was also reported as highly specific for RA. By 1998, van Venrooij's group was able to demonstrate that the common denominator for several of these autoantibody systems was the reactivity against citrullinated peptides [23]. APF and AKA are related to the citrullinated protein filaggrin whereas $\mathrm{Sa}$ is related to citrullinated vimentin [23]. More recently, the term 'citrullinome' was used referring to the whole array of citrullinated proteins, 53 in all at this time, identified in sera and synovial fluid of RA patients $[16,23]$. ACPA recognize peptides and proteins containing citrulline, a non-standard amino acid generated by the posttranslational modification of arginine by peptidylarginine deiminase enzymes, in a calcium-dependent process known as citrullination (Fig. 2a) $[11,16]$. Post-translationally modified proteins have been described to be particularly capable of inducing immunological tolerance breakdown and autoantibody response. These modifications are critical for protein structure and biological function [24]. Citrullination occurs during many biologic processes, such as inflammation, apoptosis and keratinization. ACPA are produced by plasma cells in RA joints, and the citrullination of proteins during the inflammatory process seems to play a role in triggering cognate autoantibody production. Several citrullinated proteins can be found in RA synovium, however, fibrin is the major citrullinated protein in RA joint $[11,16]$.

ACPA are detected in approximately $2 / 3$ of RA patients with a diagnostic specificity of $98 \%$ [16]. In the natural history of RA, ACPA immune response starts several years before diagnosis of the disease and the onset of symptoms, but in a restricted manner with low antibody titers and limited peptide reactivity. Fine specificity and epitope spreading, increase in titer, isotype switching and maturation of response gradually occur along the years towards the clinical onset of disease and tend to persist in the majority of patients. This evolution is associated with increase in the diversity of antibody structure that may result in the activation of more immune effector mechanisms [25].

Importantly, the rate of seroconversion of ACPA-negative early inflammatory arthritis (or early RA) to ACPA-positive disease is very low, thus suggesting that repeated testing during follow up may not have an added value [26]. In arthralgia patients, the development of arthritis is predicted not only by the presence of ACPA, but also by their levels [27]. High titer ACPA is also associated with the recognition of several citrullinated epitopes. Patients with arthralgia who

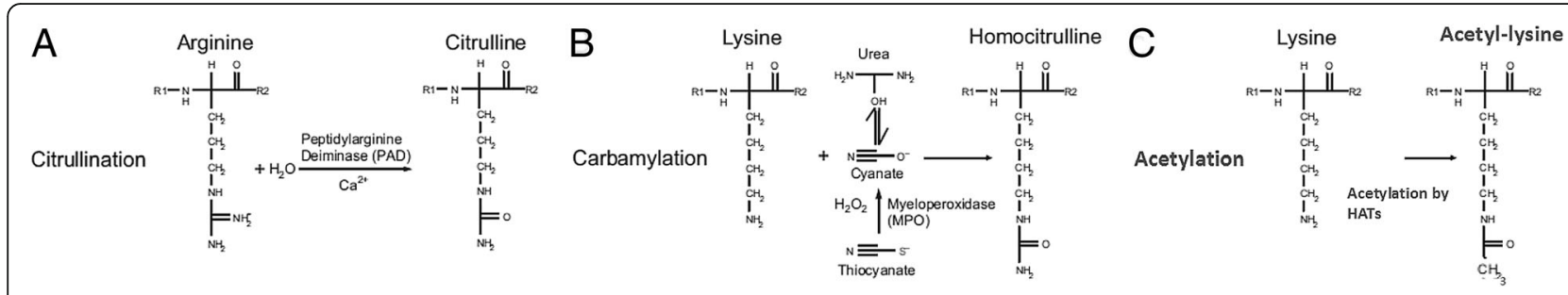

Fig. 2 Schematic illustration of post-translationally modified proteins: a Citrullination, (b) Carbamylation, and (c) Acetylation 
have an extended ACPA repertoire are at higher risk of developing arthritis [27]. These findings are consistent with the notion that a broader ACPA recognition profile is associated with higher probability for the transition towards disease $[25,27]$.

ACPA can be present in different isotypes: IgG, IgA, IgM and IgE. In ACPA-positive patients with RA, IgG1 and IgG4 ACPA are usually present in almost $99 \%$ of patients, followed by IgG2 and IG3 in 80 and $60 \%$ of patients, respectively. IgM and IgA are present in around $60 \%$ of patients $[12,13,25]$. The fine specificity and isotype usage of ACPA in health and disease differs. Healthy family members of patients with RA have fewer ACPA isotypes than their relatives with the disease $[10,14]$. The ACPA isotype distribution does not seem to significantly expand anymore during disease progression from undifferentiated arthritis to RA, indicating that most of the expansion of ACPA isotype happens before the onset of arthritis. Indeed, ACPA-positive patients with symptoms of RA for less than 12 weeks show no difference in the specificity and isotype repertoire of their ACPA response compared with patients with longer symptom duration [25]. Importantly, the number of isotypes used by ACPA also associate with RA prognosis, as the magnitude of the ACPA isotype profile at baseline reflects the risk of future radiographic damage, showing an odds ratio of 1.4-fold increase for every additional isotype [24, 28].

In patients with RA, ACPA do not show avidity maturation during longitudinal follow up. In fact, even in patients who displayed extensive isotype switching, ACPA avidity was relatively low. This data shows that there are intrinsic differences between the dynamics of development of RA-specific autoantibodies and protective antibodies against pathogens [25].

\section{ACPA testing}

The first enzyme-linked immunosorbent assay (ELISA) using citrullinated peptides (derived from filaggrin epitopes) was developed in 1988, which within 2 years was followed by the development of an ELISA based on artificial cyclic citrullinated peptides (CCP) [25]. The CCP2 assay, the first commercial version of this test, became available in 2002 and allowed the widespread routine testing for antibodies directed against citrullinated epitopes as a biomarker for RA [25]. The CCP2 peptides ensure the detection of a broad range of antibodies to citrullinated host proteins and proved to be extremely specific (98\%) for RA, displaying a significantly higher specificity in comparison with the IgM RF [25]. The overall sensitivity of anti-CCP2 assays is similar to that of RF (60-80\%), but anti-CCP2 antibody is positive in $20-30 \%$ of RF seronegative patients [29].

Other assays for detecting ACPA were subsequently developed, such as CCP3 and MCV (Mutated Citrullinated
Vimentin), with slight differences in terms of specificity and sensitivity [25]. CCP3 is based on ELISA using a collection of citrullinated peptides by a manufacturer distinct from the one that developed CCP2. Citrullinated vimentin has been identified as potential genuine autoantigen in the pathophysiology of RA, what has triggered the development of an ELISA assay for the detection of antibodies directed against mutated citrullinated vimentin (anti-MCV) [30]. Anti-MCV is a further development of the protocol for detecting antibodies to naturally citrullinated vimentin ( $\mathrm{Sa}$ antigen) [31]. Studies show that anti-MCV assay does not appear to provide additional diagnostic performance over anti-CCP in RA patients [30]. However, when patients with early RA are compared with healthy controls, it has been reported that analysis of anti-MCV yields greater sensitivity and unchanged specificity as compared with anti-CCP2. Besides, anti-MCV appears to perform better than anti-CCP2 in identifying poor radiographic prognosis in patients with early RA [30].

There is still conflicting data regarding the diagnostic and monitoring value of anti-Sa antibodies. A recent study aiming to evaluate the prevalence and diagnostic significance of anti-Sa compared with anti-CCP2 did not demonstrate any additional diagnostic value of the anti-Sa autoantibody in comparison to the anti-CCP2 [31]. Despite high specificity (92-98\%), anti-Sa antibodies showed a low diagnostic sensitivity (between 31 and 44\%) [31]. However, it has also been suggested that the combined application of anti-CCP 2 and anti-Sa tests can improve the laboratory diagnosis of early RA, with a high specificity (99.4\%), albeit with low sensitivity (50\%) [32]. It has been shown that, the recently described anti-CarP antibody correlates with anti-Sa antibodies in RA. The association of anti-CarP with anti-Sa antibodies could not be explained by cross-reactivity and this finding is interesting since both autoantibodies are associated with radiographic progression. Therefore, co-expression of anti-CarP and anti-Sa may be confounding these reports. On the other hand, presence of both of them may be associated with improved ability to predict erosive RA [33]. The CCP3 was developed by a distinct company from anti-CCP2 and does not represent a traditional technical upgrade. Despite some controversy in the literature, there seems to be no argument for superiority of one over the other one. One study showed a significantly higher sensitivity for antiCCP3 in testing RF-negative RA as well as the total RA population [29]. Recently, differences in the test performances accordingly to the moment of the natural history of RA were reported: in patients with established RA, CCP2 was more specific, whereas in subjects with undifferentiated inflammatory arthritis, CCP3 had a higher predictive value for development of RA [9]. 


\section{Role in pathogenesis}

The identification of ACPA has been a major breakthrough in the advancement of the understanding of the pathogenesis in RA. ACPA-positive and ACPA-negative disease have been shown to be associated with different genetic and environmental background, and therefore, different pathophysiological mechanisms should underlie these two separate disease subsets [25].

RA patients exhibit an abnormal humoral response to citrullinated proteins, which are expressed in any form of inflammation, in the synovium or elsewhere [25]. Normally, citrullinated proteins are regularly degraded and do not elicit any relevant humoral reaction of the immune system, therefore the presence of citrullinated proteins per se will not necessarily lead to chronic inflammation [3]. Citrullination has been reported to be a process present in a wide range of inflammatory tissues, suggesting that this is an inflammationassociated phenomenon that should be normally tolerated by the immune system. In fact, it has been widely demonstrated that the presence of citrullinated proteins is not specific for rheumatoid synovial tissue; rather, they can be observed in synovial tissue of patients with other arthropathies and in tonsils from patients with chronic tonsillitis, multiple sclerosis and type 1 diabetes [9].

Citrullination also seems to be implicated in several physiological processes, such as cell death pathways, in which intracellular calcium concentration raises to higher levels than in physiologic conditions, activating peptidylarginine deiminases (PAD) enzymes during apoptosis.

Immune cells infiltrating the inflamed tissue contain PAD enzymes. PAD activation due to high intracellular calcium concentration during cell death would promote citrullination of target antigens. Normally, the generated apoptotic bodies are rapidly removed by phagocytes, preventing inflammatory reactions. Any dysregulation of apoptosis or an ineffective clearance of apoptotic cell remnants may be involved in the breakdown of self-tolerance due to accumulation of dying cells and consequent accessibility of intra-cellular antigens. This scenario would promote the meeting of citrullinated proteins with the immune system leading to autoantibody generation in genetically predisposed individuals. This will ultimately result in immune complex formation, followed by upregulation of proinflammatory cytokines, which are regarded as the driving force of the chronic inflammation that is typical of RA (Fig. 3) [9].

Genetic factors, such as the HLA-DRB1 SE alleles, environmental factors, such as smoking and hormone levels, and the possible contribution of bacterial PAD enzymes might participate in this mechanism [5-7]. The development of an autoimmune response against citrullinated epitopes is facilitated by specific genetic predisposition. The presence of particular HLADRB1 alleles ("shared epitope"-SE) in RA patients contributes to the development of anti-CCP antibodies $[3,7,8]$. Carriage of SE alleles or the R620W allele of the general autoimmunity marker tyrosine phosphatase non-receptor type 22 (PTPN22) in smokers increases susceptibility to RA since SE related HLA binds citrullinated peptides more strongly and the R620W PTPN22 allele stimulates an exaggerated $\mathrm{T}$ cell response [5]. This $\mathrm{T}$ cell response may drive increased autoantibody production by B-cells, including ACPA [5, 7, 9]. Intriguingly, the strong association between SE-encoding HLA-DRB1 alleles and RA is only observed for ACPA-positive disease [6, 9].

Periodontitis is associated with increased risk for RA. The presence of periodontitis in patients with RA has been associated with seropositivity for RF and ACPA [34]. Porphyromonas gingivalis, a microbe that is the major causative agent for periodontitis, is the only prokaryotic organism expressing PAD, and can cause microbial and host protein citrullination [34]. Hypothetically, this may trigger an immunological response to citrullinated proteins in a subset of RA patients with periodontitis carrying SE alleles [9]. Just like chronic exposure to citrullinated proteins at periodontal sites could contribute to the breakdown of immune tolerance to citrullinated epitopes, chronic inflammation in the lungs from smokers may also predispose susceptible individuals to the development of ACPA and prime individuals to the development of RA [9]. The presence of ACPA before signs of inflammation in joints suggests that immunity against citrullinated proteins is initiated outside the joint [9]. Recent studies suggested that the lung is involved in the citrullination of proteins and may contribute for the generation of RA-related autoimmunity. Smoking has been shown to enhance PAD expression in bronchi-alveolar lavage cells with consequent generation of citrullinated proteins that may lead to citrulline autoimmunity in genetically susceptible RA subjects [7]. It was also shown that local production of ACPA can occur in lungs of patients with RA. Therefore, the lung might be a site of priming the immunity to citrullinated proteins (Fig. 4) [7].

Once generated, ACPA can induce damage as they activate the classical and alternative complement pathways. ACPA are also capable of triggering immune cell responses via $\mathrm{Fc}$ receptors $(\mathrm{FcR})$. Immune complexes containing ACPA and citrullinated fibrinogen have been shown to induce TNF $\alpha$ secretion via engagement of FcR on macrophages [25]. ACPA bind to osteoclast surfaces, resulting in osteoclastogenesis and bone degradation [4]. Another mechanism by which ACPA mediate pro-inflammatory action may be through neutrophil extracellular traps (NET). ACPA can enhance the formation of NET, resulting in expel of immune-stimulatory molecules together with strongly immunogenic citrullinated 


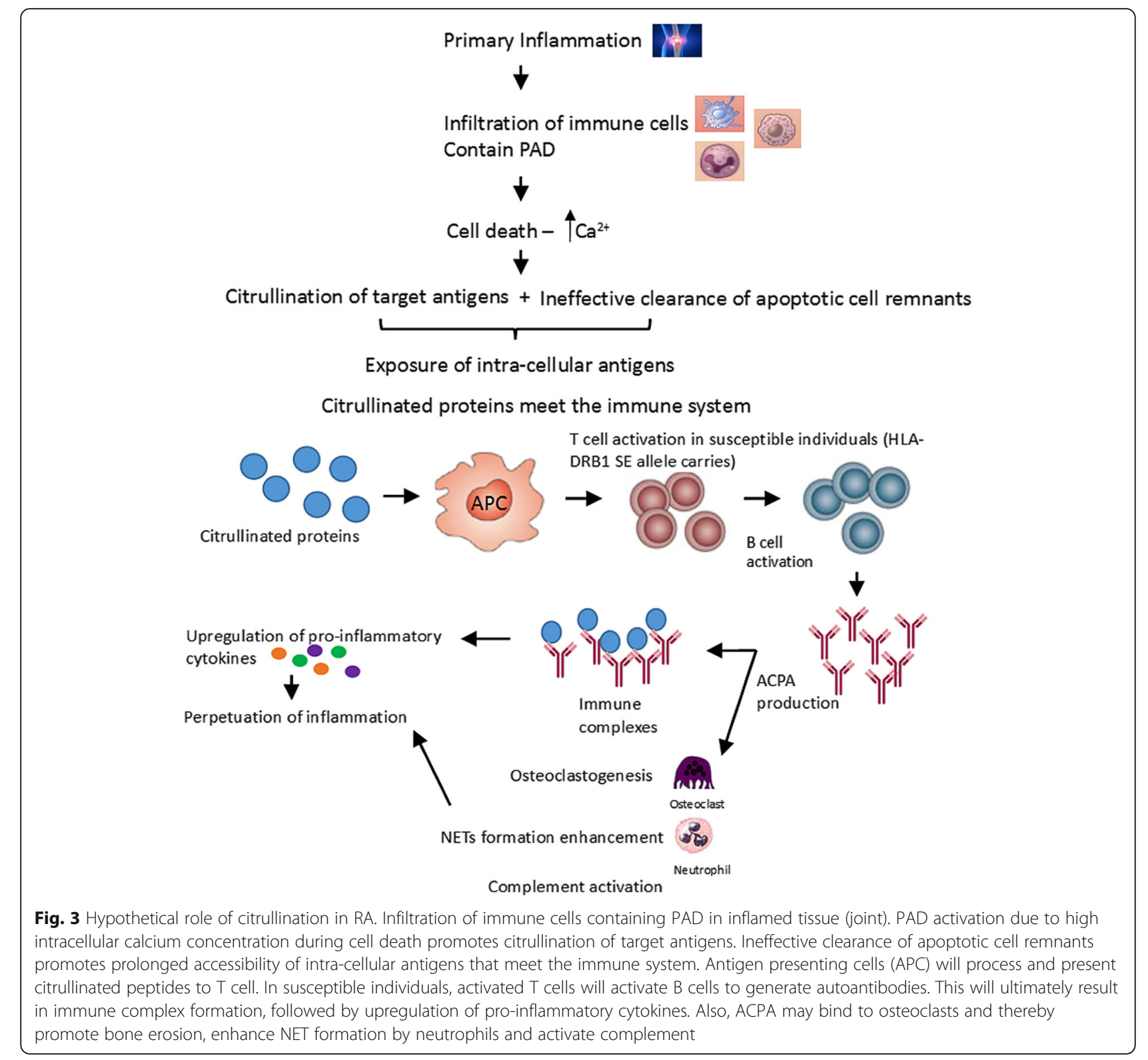

autoantigens. These observations suggest a mechanism that may promote and perpetuate disease (Fig. 3) [24, 25].

The presence of both RF and ACPA is associated with increased systemic inflammation and disease activity in RA [4]. The combined presence of IgM-RF and ACPA mediates increased pro-inflammatory cytokine production in vitro [4]. RF seems to preferentially interact with hypoglycosylated IgG and ACPA IgG is hypoglycosylated as compared with total IgG. It is suggested that IgM-RF enhances the capacity of ACPA immune complexes to stimulate macrophage cytokine production, therefore providing a mechanistic link by which RF enhances the pathogenicity of ACPA immune complexes in RA [25].

\section{Clinical relevance}

ACPA are detected in serum samples up to 14 years before onset of the first symptoms of RA and IgM-RF up to 10 years [35]. The presence of ACPA is associated with more severe joint destruction and ACPA-positive patients develop erosions earlier and more abundantly than patients without ACPA [1, 9]. It is also associated with greater disease activity and poorer remission rates [9]. In addition, the extra-articular manifestations that often determine the severity and comorbidity of RA are also closely associated with ACPA positivity [25]. Both ACPA and RF have been found to associate with cardiovascular disease and mortality in RA patients [4]. Their presence predicts progression towards RA in patients 


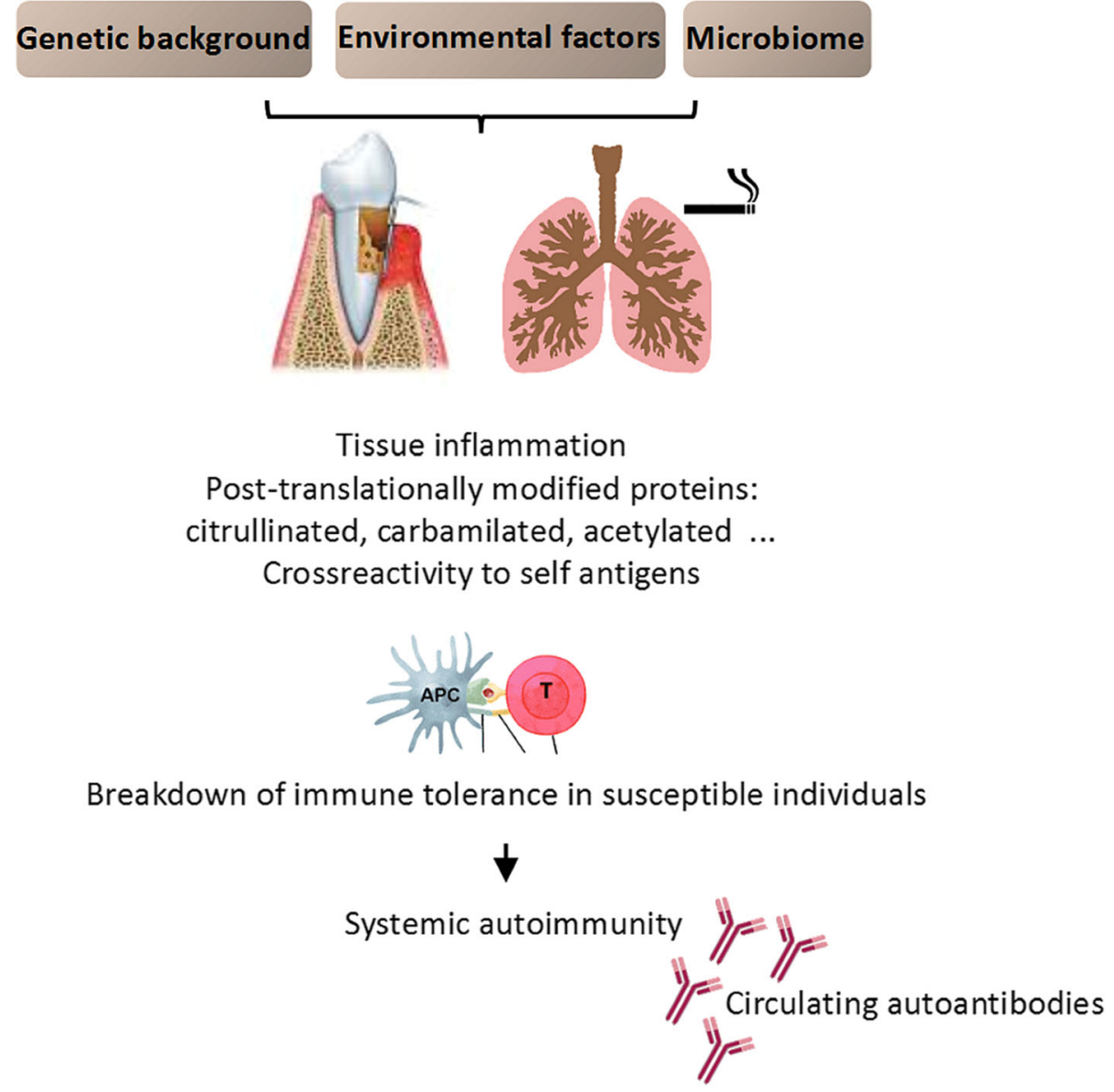

Fig. 4 Genetic, environmental factors, such as smoking and the possible occurrence of bacterial PAD enzymes lead to post-translational protein modifications in inflamed tissues. The breakdown of immune tolerance in susceptible individuals may lead to antibody formation and systemic inflammation

with undifferentiated arthritis and high levels are indicative for arthritis development in arthralgia patients [27]. ACPA positivity is also associated with the presence of RF and SE [29, 36]. Combination analysis showed independent additive effects of these three factors for high radiological risk [36]. It has been shown that anti-CCP2 has higher positive predictive value for erosive $\mathrm{RA}$ than $\mathrm{RF}$, C-reactive protein (CRP), erythrocyte sedimentation rate (ESR) or matrix metalloproteinase-3 (MMP-3) serum levels [37]. ACPA-positive patients seem to respond better to treatment than ACPA-negative RA patients in an early phase of the disease, but achieve drug-free remission less frequently [4].

ACPA-positive patients with undifferentiated arthritis (UA) benefit from treatment with methotrexate being less likely to progress to RA, and doing so at a later time point, as compared with a placebo control group. In addition, fewer patients under methotrexate show radiographic progression over 18 months. In contrast, no effect of methotrexate therapy on progression to RA was observed in the
ACPA-negative group [38]. ACPA-positive patients, with low and intermediate pre-treatment levels of ACPA respond better to methotrexate treatment in recent-onset cohorts, whereas high levels are associated with an insufficient response. Therefore, in patients with high ACPA levels, methotrexate monotherapy might be insufficient [39]. In the BeSt study, ACPA-positive patients initially treated with DMARD monotherapy had greater radiographic joint destruction after 2 years than ACPA-negative patients. However, when patients were treated initially with combination therapy (DMARD plus anti-TNF biologicals), no difference regarding joint destruction was observed between ACPA-positive and ACPA-negative patients. These observations suggest that ACPA-positive patients, especially those with high titer ACPA, require an aggressive initial approach in order to prevent radiographic progression [25].

ACPA titers can decrease over the course of disease when patients have a good response to therapy. DMARD induce a reduction of $25 \%$ or more in ACPA titers in half of the patients over the course of treatment [40]. 
Regarding response to TNF inhibitors, one study has shown that the presence of ACPA was associated with reduced response to those agents [41]. Other studies show that response to TNF inhibitors is associated with lower ACPA baseline titers and that there is around 30\% reduction of serum ACPA titers after anti-TNF treatment [40]. However, several other reports showed little or no effect of anti-TNF therapy on ACPA titers [40]. Other immunobiologicals, such as abatacept, reduce $\mathrm{CD}^{+} 0^{+} \mathrm{B}$ cells in the synovial membrane of RA patients and the production of IL-2, IL-17, IL-22 in ACPA-positive but not in ACPA-negative RA patients [42, 43]. In these studies, anti-CCP2 positivity has been associated with EULAR response, suggesting that abatacept is more efficacious in ACPA-positive RA patients. Rituximab is associated with good to moderate EULAR response in ACPA-positive patients or in patients with high ACPA levels. Anti-CCP2 antibody levels after rituximab therapy present a more pronounced fall in responders relative to non-responders [44]. These data indicate that ACPA status may be relevant for treatment decisions in RA and support the hypothesis that RA can be classified into two different disease subsets: ACPA-positive and ACPA-negative RA [25].

\section{Anti-carbamylated protein antibodies (anti-CarP) Characteristics}

Recently, a new autoantibody system has been described in RA, characterized by antibodies against carbamylated proteins, i.e., proteins that contain homocitrulline residues (anti-CarP antibodies). Antibodies in the serum of RA patients can discriminate citrullinated and carbamylated antigens and, therefore, this antibody system is independent from ACPA. In fact, anti-CarP antibodies may be detected in ACPA-negative patients and vice-versa [4, 45].

Carbamylation is defined as a post-translational modification in which a positively charged amino acid is replaced by a neutral amino acid. The most common carbamylation process refers to the conversion of lysine into homocitrulline. The chemical structure of homocitrulline resembles citrulline. Homocitrulline is one methylene group longer than citrulline (Fig. 2b) [40, 45, 46]. In contrast to citrullination, carbamylation is a non-enzymatic chemical reaction involving cyanate in the conversion of lysine into homocitrulline. Cyanate is naturally present in the several body fluids and in equilibrium with urea [45]. Under physiological conditions, the cyanate concentration is too low to allow extensive carbamylation of proteins. However, several conditions such as renal disease, inflammation and smoking can shift the balance towards predominance of cyanate over urea. In renal failure, the urea concentration increases, resulting in extensive carbamylation of proteins [47]. Smoking also increases the cyanate concentration and can enhance carbamylation. However, most carbamylation is believed to take place under inflammatory conditions, when myeloperoxidase (MPO) is released from neutrophils, converting thiocyanate to cyanate, an essential driver for carbamylation [45]. As a consequence of excess carbamylation, protein and cellular dysfunction may occur, leading to systemic effects [45]. Decreased functional activity upon carbamylation has been reported for several enzymes and hormones. In susceptible individuals, extensive carbamylation will provide the trigger for the development an autoimmune response directed against carbamylated proteins (Fig. 5) [45].

\section{Pathogenesis}

In animal model, it has been shown that carbamylated proteins can trigger primary immune responses, inducing chemotaxis, $\mathrm{T}$ cell activation and antibody production, and subsequently, the production of IFN- $\gamma$, IL-10 and IL-17. The activation of $\mathrm{T}$ cells added to a strong antibody response will enable the recognition of carbamylated and citrullinated peptides within the joints, which may contribute to the development of erosive arthritis. Carbamylated and citrullinated peptides complement each other in the generation of the autoimmune response. The immune-activating effects of carbamylation enhance the arthritogenic properties of citrullinated peptides, therefore providing a novel mechanism for the pathogenesis of autoimmune arthritis [46].

\section{Clinical relevance}

Anti-CarP antibodies are detected in up to $45 \%$ of RA patients (45\% IgG and 43\% IgA anti-CarP). Notably, anti-CarP antibodies may occur in 16-30\% of ACPA-negative patients (16\% IgG and 30\% IgA anti-CarP) [48]. Anti-CarP IgG antibodies seem to be associated with a more severe radiological progression in ACPA-negative RA, indicating that anti-CarP antibodies are a unique and relevant serological marker for ACPA-negative patients [48]. Recently, the presence of anti-CarP antibodies was associated also with higher disease activity and significantly more disability over time in patients with rheumatoid arthritis. Statistically significant associations were seen not only in ACPA-positive but also in ACPA-negative patients [45].

These autoantibodies can be detected more than 10 years before disease onset, at the same time of ACPA and before IgM-RF [4, 49]. The presence of anti-CarP antibodies of patients with arthralgia predicts the development of RA independently of ACPA [49]. Therefore, anti-CarP antibodies might be a useful biomarker to identify ACPA-negative "pre-RA" patients and newly diagnosed RA patients who require early and aggressive clinical intervention $[45,49]$.

The high specificity of anti-CarP antibodies for RA was suggested as these antibodies were not found in patients with other inflammatory rheumatic conditions or in normal healthy individuals [50]. However, one study 


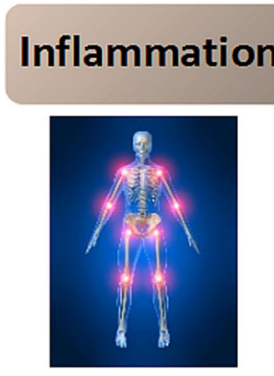

\section{Smoking Renal Disease}
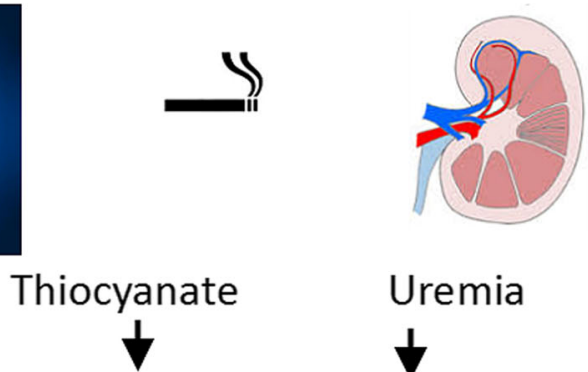

Uremia

Cyanate
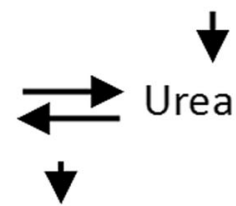

Urea

\section{Carbamylation}

Protein dysfunction

\section{Cellular dysfunction}

Systemic effects
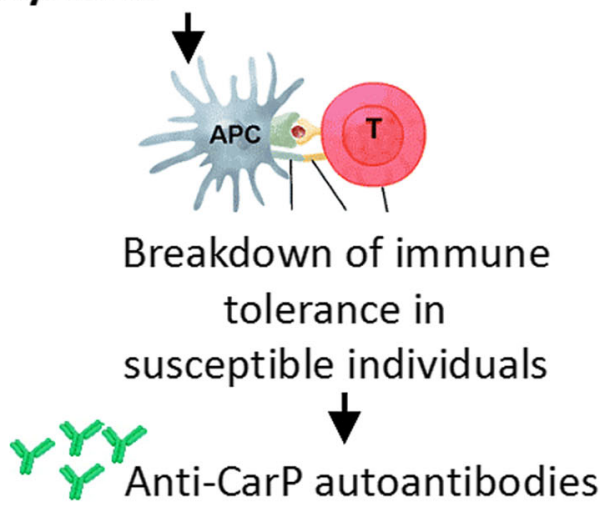

Fig. 5 Schematic representation of causes and consequences of carbamylation. Inflammation, smoking and renal disease may shift the balance towards predominance of cyanate over urea, allowing carbamylation. As a consequence of excess carbamylation, protein and cellular dysfunction may occur, leading to systemic effects. In susceptible individuals, extensive carbamylation will provide the trigger for the development an autoimmune response directed against carbamylated proteins

showed that anti-CarP antibodies are detectable in the serum of patients with active PsA and correlations between anti-CarP levels and disease activity were observed in polyarthritis patients that were negative for ACPA and RF. If confirmed, anti-CarP may be considered as the first evidence of the presence of autoantibodies in PsA [51]. Anti-CarP antibodies can also be found in juvenile idiopathic arthritis (JIA) patients. It has been shown that $16.7 \%$ of JIA patients are positive for anti-CarP, whereas only $6.4 \%$ are positive for ACPA and $8.1 \%$ for IgM-RF [52]. Genetic and environmental associations have not yet been investigated thoroughly, but one study showed that there were no significant associations between anti-CarP antibodies and smoking, PTPN22 alleles or HLA-DRB1, with the exception of the association identified for HLA-DRB1*03.. The lack of association with SE-HLA alleles may indicate a different biological mechanism for the formation of anti-CarP antibodies in comparison with the development of
ACPA and may represent an opportunity to identify additional molecular pathways involved in RA pathophysiology [53].

\section{Anti-PAD4 antibodies \\ Characteristics and pathogenesis}

PAD4 is a calcium dependent peptidylarginine deiminidase, one of the proteins that are responsible for the conversion of arginine into citrulline [4]. It has been recently demonstrated that PAD4 may undergo auto-citrullination, a process that might inactivate the enzyme as a mechanism of control. PAD4 citrullination modifies the structure of the enzyme, increasing its recognition by human autoantibodies [9, 54]. In fact, autoantibodies directed against PAD4 have indeed been identified in RA patients [4]. These antibodies not only target but also activate PAD, increasing the catalytic efficiency of the enzyme by decreasing its requirement for calcium [55]. Anti-PAD4 antibodies have 
been reported to have predictive and prognostic value in RA patients [9].

PAD is found also in Porphyromonas Gingivalis (PPAD) However, recent data showed that PPAD expressed by $P$ gingivalis is not citrullinated and PPAD citrullination is not recognized by anti-PAD antibodies in RA. Besides, anti-PPAD antibodies were not associated with ACPA levels and disease activity in RA and seem to have a protective role for periodontitis development in RA patients [9].

\section{Clinical relevance}

Anti-PAD4 antibodies are present in $22-45 \%$ of RA patients and can also be detected in 14\% of SLE patients, but spondyloarthritis patients do not seem to have these autoantibodies [4]. It has low specificity for RA diagnosis (< 50\%) [4]. Anti-PAD4 antibodies are almost exclusively found in people with established RA and have been associated with severe disease in these patients [4]. These antibodies are associated with the presence of ACPA and are usually detected after ACPA appearance [56].

A specific group of anti-PAD4 antibodies cross-reacts with anti-PAD3 antibodies. This subset increases the catalytic capacity of PAD4 by decreasing its calcium requirement for citrullination. These antibodies are present in 12 to $18 \%$ of RA patients and its reactivity has been found only in anti-PAD4 positive RA patients. Importantly, these cross-reactive antibodies were associated with radiographic damage severity [55]. In addition, the prevalence and extent of interstitial lung disease was found to be higher among RA patients with anti-PAD3/4 cross-reactive antibodies [57]. Therefore, anti-PAD3/4 antibodies may serve as biomarker for disease prognosis, despite being detected at low frequency in RA patients [4].

\section{Anti-BRAF antibodies}

\section{Characteristics and pathogenesis}

BRAF (v raf murine sarcoma viral oncogene homolog B1) catalytic domain is a serine-threonine kinase that regulates the mitogen-activated protein kinase (MAPK) signaling pathway implicated in the production of pro-inflammatory cytokines. Anti-BRAF autoantibodies activate BRAF kinase activity, which may lead to production of pro-inflammatory cytokines and joint inflammation [54]. Anti-BRAF antibodies are present in $21-32 \%$ of RA patients.

\section{Clinical relevance}

These autoantibodies are present also in SLE and primary Sjögren's syndrome in a similar frequency to RA, and also in $4 \%$ of ankylosing spondylitis patients and $6 \%$ of healthy individuals $[4,54]$. Although not specific for RA, anti-BRAF may be an interesting new autoantibody to identify ACPA-negative RA patients since $30 \%$ of anti-CCP2 negative RA patients were positive for anti-BRAF antibodies [54].

\section{Other autoantibodies}

\section{Anti-RA-33 or anti-hnRNP A2 antibodies}

RA-33 is an intracellular molecule that binds to the heterogeneous nuclear protein (hnRNP) A2, a part of the splicosome. Anti-RA-33 antinuclear antibodies are present in one third of RA patients. The reported frequency of anti-RA-33 antibodies is 13\% in ACPA/RF-negative patients and $9 \%$ in non-RA patients. Anti-RA33 antibodies may not be useful as a clinical biomarker for diagnosing RA, however anti-RA33-positive patients seem to show a less severe disease so that these antibodies might serve as a prognostic marker for less aggressive disease [4].

\section{Anti-malondialdehyde and anti-malondialdehyde acetaldehyde antibodies}

Post-translational modifications due to lipid peroxidation can result in the presence of malondialdehyde (MDA) and malondialdehyde-acetaldehyde (MAA)-adducts. It was found that both MAA adducts and antibodies directed against these adducts were increased in the serum of RA patients, and a positive correlation between the presence of ACPA and anti-MAA antibodies was observed [58]. However, anti-MAA antibodies have also been detected in chronic liver diseases and in type 2 diabetes [59]. Antibodies against MDA-adducts, especially MDA-LDL, were found to associate with cardiovascular problems in RA patients.

\section{Anti-acetylated peptide antibodies}

Acetylation is a reversible enzymatic process where acetyl groups are added to free amines of lysine residues by Lys acetyltransferases (KAT) [60]. Acetylated lysine resembles homocitrulline, but the side chain terminal amine is replaced by a methyl moiety in acetylate lysine (Fig. 2c) [61].

Protein lysine acetylation is a key post-translational modification in cellular regulation, especially in histones and nuclear transcription regulators. Acetylation of cytoplasmic proteins regulates metabolic pathways and enzymatic functions [62]. IgG and IgA antibodies against acetylated vimentin peptides were detected in $35 \%$ of patients with early arthritis. However, data showed that anti-acetylated vimentin antibodies are relatively poor for predicting the development of anti-ACPA-negative RA. Their presence and frequency in established RA and their role in predicting disease severity and other clinically relevant outcomes in patients with RA remain to be established [61].

\section{Anti-oxidized protein antibodies in RA}

Several data suggest a role for oxidative stress in the pathogenesis of RA. Reactive oxygen species (ROS have been identified in the synovial fluid of $90 \%$ of RA patients [40]. Studies show that type II collagen (CII) post-translationally modified by ROS (ROS-CII) is present in the inflamed joints [63]. High titer anti-ROS-CII reactivity was observed 
in early RA regardless of ACPA status (93.8\% in ACPApositive patients and $91.6 \%$ in ACPA-negative patients). The sensitivity and specificity of anti-ROS-CII antibodies in early RA was 92 and 98\%, respectively [40]. Anti-ROS-CII activity has not been detected in the serum of patients with other inflammatory diseases [40]. ROS-CII reactivity was lower in RA patients after their first DMARD treatment and this was associated with good response: $7.6 \%$ in serum samples of responders and $58.3 \%$ of serum samples in non-responders. ${ }^{191}$

Other less well-studied autoantibodies have been reported in RA. Autoantibodies against transthyretin, a hormone carrier, were found to be increased in RA patients when compared to healthy controls [4]. Antibodies against the hinge region of immunoglobulins, anti-hinge antibodies (AHA), have been reported in approximately 15-20\% of RA patients. Despite low sensitivity, they seem to have high specificity for RA. ACPA antibodies may be fragmented by inflammation-associated proteases in the hinge region, creating novel epitopes that can be recognized by the immune system, resulting in AHA. These autoantibodies could modulate arthritis by binding to fragmented autoantibodies in the inflamed joint, which may lead to exacerbation of disease [64].

\section{Conclusion}

Several RA-associated autoantibody systems have been identified and many of these autoantibodies recognize post translationally modified proteins, indicating the immunogenicity of such proteins for human B cells.

RF and ACPA are the two most remarkable autoantibodies in RA and provide different clinical and pathophysiological information. ACPA exhibit high sensitivity with the highest predictive value for RA development and severity. These autoantibodies enabled the stratification of RA in ACPA-positive and ACPA-negative disease phenotype, with different genetic and environmental contribution factors. In addition, ACPA status predicts response to therapy. However, despite the diagnostic value of RF and ACPA, more serological markers are needed in order to improve early diagnosis and treatment of the patients as well as to lead to a better understanding of the molecular pathways involved in RA.

Recently, the identification of anti-CarP antibodies, also present in the serum of RF-negative/ACPA-negative RA patients filled another gap in the seronegative RA spectrum, and indicated further heterogeneity among RA patients. Hopefully, the progressive characterization of a comprehensive array of novel autoantibody systems in RA will provide unique pathogenic insights of relevance for the development of diagnostic and prognostic approaches compatible with an effective personalized medicine.
Acknowledgements

Not aplicable

Funding

Not aplicable

Availability of data and materials

Not aplicable

Authors' contributions

All authors read and approved the final manuscript.

Ethics approval and consent to participate

Aprovação CEP 44990815.5.0000.5505.

\section{Competing interests}

The authors declare that they have no competing interests.

\section{Publisher's Note}

Springer Nature remains neutral with regard to jurisdictional claims in published maps and institutional affiliations.

Received: 7 November 2018 Accepted: 29 November 2018

Published online: 17 January 2019

\section{References}

1. Moeez S, John P, Bhatti A. Anti-citrullinated protein antibodies: role in pathogenesis of RA and potential as a diagnostic tool. Rheumatol Int. 2013;33(7):1669-73.

2. Szodoray P, Szabó Z, Kapitány A, Gyetvai A, Lakos G, Szántó S, et al. Anti-citrullinated protein/peptide autoantibodies in association with genetic and environmental factors as indicators of disease outcome in rheumatoid arthritis. Autoimmun Rev. 2010;9(3):140-3.

3. Farid SS, Azizi G, Mirshafiey A. Anti-citrullinated protein antibodies and their clinical utility in rheumatoid arthritis. Inter J Rheum Dis. 2013;16(4):379-86.

4. Verheul MK, Fearon U, Trouw LA, Veale DJ. Biomarkers for rheumatoid and psoriatic arthritis. Clin Immunol. 2015;161(1):2-10.

5. Klareskog L, Malmström V, Lundberg K, Padyukov L, Alfredsson L. Smoking, citrullination and genetic variability in the immunopathogenesis of rheumatoid arthritis. Semin Immunol. 2011;23(2):92-8.

6. Imboden JB. The immunopathogenesis of rheumatoid arthritis. Annu Rev Pathol. 2009:4:417-34.

7. Kallberg H, Padyukov L, Plenge RM, Ronnelid J, Gregersen PK, van der Helmvan Mil AH, et al. Gene-gene and gene-environment interactions involving HLA-DRB1, PTPN22, and smoking in two subsets of rheumatoid arthritis. Am J Hum Genet. 2007:80(5):867-75.

8. Aletaha D, Neogi T, Silman AJ, Funovits J, Felson DT, Bingham CO 3rd, et al. Rheumatoid arthritis classification criteria: an American College of Rheumatology/European league against rheumatism collaborative initiative. Arthritis Rheum. 2010;62(9):2569-81.

9. Valesini G, Gerardi MC, lannuccelli C, Pacucci VA, Pendolino M, Shoenfeld Y. Citrullination and autoimmunity. Autoimmun Rev. 2015;14(6):490-7.

10. Ingegnoli F, Castelli R, Gualtierotti R. Rheumatoid factors: clinical applications. Dis Markers. 2013;35(6):727-34

11. Moura RA, Graca L, Fonseca JE. To B or not to B the conductor of rheumatoid arthritis orchestra. Clin Rev Allergy Immunol. 2012;43(3):281-91.

12. Nishimura K, Sugiyama D, Kogata Y, Tsuji G, Nakazawa T, Kawano S, et al. Meta-analysis: diagnostic accuracy of anti-cyclic citrullinated peptide antibody and rheumatoid factor for rheumatoid arthritis. Ann Intern Med. 2007;146(11):797-808.

13. Jónsson T, Steinsson K, Jónsson H, Geirsson AJ, Thorsteinsson J, Valdimarsson $\mathrm{H}$. Combined elevation of IgM and IgA rheumatoid factor has high diagnostic specificity for rheumatoid arthritis. Rheumatol Int. 1998; 18(3):119-22.

14. Nielsen SF, Bojesen SE, Schnohr P, Nordestgaard BG. Elevated rheumatoid factor and long term risk of rheumatoid arthritis: a prospective cohort study. BMJ. 2012;345:e5244.

15. Edwards JC, Cambridge G. Rheumatoid arthritis: the predictable effect of small immune complexes in which antibody is also antigen. $\mathrm{Br} J$ Rheumatol. 1998;37(2):126-30 
16. Song YW, Kang EH. Autoantibodies in rheumatoid arthritis: rheumatoid factors and anticitrullinated protein antibodies. QJM. 2010;103(3):139-46.

17. Jónsson T, Arinbjarnarson S, Thorsteinsson J, Steinsson K, Geirsson AJ, Jónsson $\mathrm{H}$, et al. Raised lgA rheumatoid factor (RF) but not lgM RF or lgG $\mathrm{RF}$ is associated with extra-articular manifestations in rheumatoid arthritis. Scand J Rheumatol. 1995;24(6):372-5.

18. Sokolove J, Johnson DS, Lahey LJ, Wagner CA, Cheng D, Thiele GM, et al. Rheumatoid factor as a potentiator of anti-citrullinated protein antibodymediated inflammation in rheumatoid arthritis. Arthritis Rheumatol. 2014; 66(4):813-21.

19. Bos WH, Bartelds GM, Wolbink GJ, de Koning MH, van de Stadt RJ, van Schaardenburg D, et al. Differential response of the rheumatoid factor and anticitrullinated protein antibodies during adalimumab treatment in patients with rheumatoid arthritis. J Rheumatol. 2008;35(10):1972-7.

20. Bobbio-Pallavicini F, Caporali R, Alpini C, Moratti R, Montecucco C. Predictive value of antibodies to citrullinated peptides and rheumatoid factors in antiTNF-alpha treated patients. Ann N Y Acad Sci. 2007:1109:287-95.

21. Klaasen R, Cantaert T, Wijbrandts CA, Teitsma C, Gerlag DM, Out TA, et al. The value of rheumatoid factor and anti-citrullinated protein antibodies as predictors of response to infliximab in rheumatoid arthritis: an exploratory study. Rheumatology (Oxford). 2011;50(8):1487-93.

22. Maneiro RJ, Salgado E, Carmona L, Gomez-Reino JJ. Rheumatoid factor as predictor of response to abatacept, rituximab and tocilizumab in rheumatoid arthritis: systematic review and meta-analysis. Semin Arthritis Rheum. 2013;43(1):9-17.

23. van Beers JJ, Schwarte CM, Stammen-Vogelzangs J, Oosterink E, Božič B, Pruijn GJ. The rheumatoid arthritis synovial fluid citrullinome reveals novel

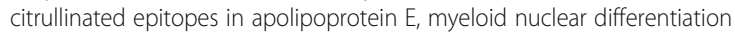
antigen, and $\beta$-actin. Arthritis Rheum. 2013;65(1):69-80.

24. Bax M, Huizinga TW, Toes RE. The pathogenic potential of autoreactive antibodies in rheumatoid arthritis. Semin Immunopathol. 2014;36(3):313-25.

25. Willemze A, Trouw LA, Toes RE, Huizinga TW. The influence of ACPA status and characteristics on the course of RA. Nat Rev Rheumatol. 2012;8(3):144-52.

26. Barra L, Pope J, Bessette L, Haraoui B, Bykerk V. Lack of seroconversion of rheumatoid factor and anti-cyclic citrullinated peptide in patients with early inflammatory arthritis: a systematic literature review. Rheumatology (Oxford). 2011;50(2):311-6.

27. van de Stadt LA, van der Horst AR, de Koning MH, Bos WH, Wolbink GJ, van de Stadt RJ, et al. The extent of the anti-citrullinated protein antibody repertoire is associated with arthritis development in patients with seropositive arthralgia. Ann Rheum Dis. 2011;70(1):128-33.

28. van der Woude D, Syversen SW, van der Voort El, Verpoort KN, Goll GL, van der Linden MP, et al. The ACPA isotype profile reflects long-term radiographic progression in rheumatoid arthritis. Ann Rheum Dis. 2010;69(6):1110-6.

29. Szekanecz Z, Szabó Z, Zeher M, Soós L, Dankó K, Horváth I, et al. Superior performance of the CCP3.1 test compared to CCP2 and MCV in the rheumatoid factor-negative RA population. Immunol Res. 2013;56(2-3):439-43.

30. Bartoloni E, Alunno A, Bistoni O, Bizzaro N, Migliorini P, Morozzi G, et al. Diagnostic value of anti-mutated citrullinated vimentin in comparison to anti-cyclic citrullinated peptide and anti-viral citrullinated peptide 2 antibodies in rheumatoid arthritis: an Italian multicentric study and review of the literature. Autoimmun Rev. 2012;11(11):815-20.

31. Iwaszkiewicz C, Puszczewicz M, Białkowska-Puszczewicz G. Diagnostic value of the anti-Sa antibody compared with the anti-cyclic citrullinated peptide antibody in rheumatoid arthritis. Int J Rheum Dis. 2015;18(1):46-51.

32. Hou YF, Sun GZ, Sun HS, Pan WP, Liu WB, Zhang CQ. Diagnostic value of anti-Sa and anticitrullinated protein antibodies in rheumatoid arthritis. J Rheumatol. 2012;39(8):1506-8.

33. Challener GJ, Jones JD, Pelzek AJ, Hamilton BJ, Boire G, de Brum-Fernandes $\mathrm{AJ}$, et al. Anti-carbamylated protein antibody levels correlate with anti-Sa (citrullinated vimentin) antibody levels in rheumatoid arthritis. J Rheumatol. 2016;43(2):273-81.

34. Dissick A, Redman RS, Jones M, Rangan BV, Reimold A, Griffiths GR, et al. Association of periodontitis with rheumatoid arthritis: a pilot study. J Periodontol. 2010;81(2):223-30

35. Nielen MM, van Schaardenburg D, Reesink HW, van de Stadt RJ, van der Horst-Bruinsma IE, de Koning $\mathrm{MH}$, et al. Specific autoantibodies precede the symptoms of rheumatoid arthritis: a study of serial measurements in blood donors. Arthritis Rheum. 2004;50(2):380-6.

36. De Rycke L, Peene I, Hoffman IE, Kruithof E, Union A, Meheus L, et al. Rheumatoid factor and anticitrullinated protein antibodies in rheumatoid arthritis: diagnostic value, associations with radiological progression rate, and extra-articular manifestations. Ann Rheum Dis. 2004:63(12):1587-93.

37. Shovman O, Gilburd B, Zandman-Goddard G, Sherer Y, Orbach H, Gerli R, et al. The diagnostic utility of anti-cyclic citrullinated peptide antibodies, matrix metalloproteinase-3, rheumatoid factor, erythrocyte sedimentation rate, and C-reactive protein in patients with erosive and non-erosive rheumatoid arthritis. Clin Dev Immunol. 2005;12(3):197-202.

38. van Dongen $H$, van Aken J, Lard LR, Visser K, Ronday HK, Hulsmans HM, et al. Efficacy of methotrexate treatment in patients with probable rheumatoid arthritis: a double-blind, randomized, placebo-controlled trial. Arthritis Rheum. 2007:56(5):1424-32.

39. Visser K, Goekoop-Ruiterman YP, de Vries-Bouwstra JK, Ronday HK, Seys PE, Kerstens PJ, et al. A matrix risk model for the prediction of rapid radiographic progression in patients with rheumatoid arthritis receiving different dynamic treatment strategies: post hoc analyses from the BeSt study. Ann Rheum Dis. 2010;69(7):1333-7.

40. Burska AN, Hunt L, Boissinot M, Strollo R, Ryan BJ, Vital E, et al. Autoantibodies to posttranslational modifications in rheumatoid arthritis. Mediat Inflamm. 2014:2014:492873.

41. Potter C, Hyrich KL, Tracey A, Lunt M, Plant D, Symmons DP, et al. Association of rheumatoid factor and anti-cyclic citrullinated peptide positivity, but not carriage of shared epitope or PTPN22 susceptibility variants, with anti-tumour necrosis factor response in rheumatoid arthritis. Ann Rheum Dis. 2009;68(1):69-74.

42. Kanbe K, Chiba J, Nakamura A. Immunohistological analysis of synovium treated with abatacept in rheumatoid arthritis. Rheumatol Int. 2013;33(7):1883-7.

43. Pieper J, Herrath J, Raghavan S, Muhammad K, Rv V, Malmström V. CTLA4-Ig (abatacept) therapy modulates $T$ cell effector functions in autoantibodypositive rheumatoid arthritis patients. BMC Immunol. 2013;14:34.

44. Gardette A, Ottaviani S, Tubach F, Roy C, Nicaise-Roland P, Palazzo E, et al. High anti-CCP antibody titres predict good response to rituximab in patients with active rheumatoid arthritis. Joint Bone Spine. 2014;81(5):416-20.

45. Shi J, van Veelen PA, Mahler M, Janssen GM, Drijfhout JW, Huizinga TW, et al. Carbamylation and antibodies against carbamylated proteins in autoimmunity and other pathologies. Autoimmun Rev. 2014;13(3):225-30.

46. Mydel P, Wang Z, Brisslert M, Hellvard A, Dahlberg LE, Hazen SL, et al. Carbamylation-dependent activation of T cells: a novel mechanism in the pathogenesis of autoimmune arthritis. J Immunol. 2010;184(12):6882-90.

47. Wynckel A, Randoux C, Millart H, Desroches C, Gillery P, Canivet E, et al. Kinetics of carbamylated haemoglobin in acute renal failure. Nephrol Dial Transplant. 2000;15(8):1183-8.

48. Shi J, Knevel R, Suwannalai P, van der Linden MP, Janssen GM, van Veelen PA, et al. Autoantibodies recognizing carbamylated proteins are present in sera of patients with rheumatoid arthritis and predict joint damage. Proc Natl Acad Sci U S A. 2011;108(42):17372-7.

49. Shi J, van de Stadt LA, Levarht EWN, Huizinga TWJ, Toes REM, Trouw LA, et al. Anti carbamylated protein antibodies (anti-CarP) are present in arthralgia patients and predict the development of rheumatoid arthritis. Ann Rheum Dis. 2013;72:A31

50. Scinocca M, Bell DA, Racapé M, Joseph R, Shaw G, McCormick JK, et al. Antihomocitrullinated fibrinogen antibodies are specific to rheumatoid arthritis and frequently bind citrullinated proteins/peptides. J Rheumatol. 2014;41(2):270-9.

51. Chimenti MS, Triggianese P, Nuccetelli M, Terracciano C, Crisanti A, Guarino MD, et al. Auto-reactions, autoimmunity and psoriatic arthritis. Autoimmun Rev. 2015;14(12):1142-6.

52. Muller PC, Anink J, Shi J, Levarht EW, Reinards $\mathrm{TH}$, Otten $\mathrm{MH}$, et al. Anticarbamylated protein (anti-CarP) antibodies are present in sera of juvenile idiopathic arthritis (JIA) patients. Ann Rheum Dis. 2013;72(12):2053-5

53. Jiang $X$, Trouw LA, van Wesemael TJ, Shi J, Bengtsson C, Källberg H, et al. Anti-CarP antibodies in two large cohorts of patients with rheumatoid arthritis and their relationship to genetic risk factors, cigarette smoking and other autoantibodies. Ann Rheum Dis. 2014;73(10):1761-8.

54. Auger I, Charpin C, Balandraud N, Martin M, Roudier J. Autoantibodies to PAD4 and BRAF in rheumatoid arthritis. Autoimmun Rev. 2012;11(11):801-3.

55. Darrah E, Giles JT, Ols ML, Bull HG, Andrade F, Rosen A. Erosive rheumatoid arthritis is associated with antibodies that activate PAD4 by increasing calcium sensitivity. Sci Transl Med. 2013;5(186):186ra65.

56. Umeda N, Matsumoto I, Kawaguchi H, Kurashima Y, Kondo Y, Tsuboi H, et al. Prevalence of soluble peptidylarginine deiminase 4 (PAD4) and anti-PAD4 antibodies in autoimmune diseases. Clin Rheumatol. 2016;35(5):1181-8.

57. Giles JT, Darrah E, Danoff S, Johnson C, Andrade F, Rosen A, Bathon JM. Association of cross-reactive antibodies targeting peptidyl-arginine 
deiminase 3 and 4 with rheumatoid arthritis-associated interstitial lung disease. PLoS One. 2014;9(6):e98794.

58. Thiele GM, Duryee MJ, Anderson DR, Klassen LW, Mohring SM, Young KA, et al. Malondialdehyde-acetaldehyde adducts and anti-malondialdehydeacetaldehyde antibodies in rheumatoid arthritis. Arthritis Rheumatol. 2015; 67(3):645-55.

59. Vehkala L, Ukkola O, Kesäniemi YA, Kähönen M, Nieminen MS, Salomaa V, et al. Plasma IgA antibody levels to malondialdehyde acetaldehyde-adducts are associated with inflammatory mediators, obesity and type 2 diabetes. Ann Med. 2013;45(8):501-10.

60. Yang XJ, Seto E. Lysine acetylation: codified crosstalk with other posttranslational modifications. Mol Cell. 2008;31(4):449-61.

61. Juarez M, Bang H, Hammar F, Reimer U, Dyke B, Sahbudin I, et al. Identification of novel antiacetylated vimentin antibodies in patients with early inflammatory arthritis. Ann Rheum Dis. 2016;75(6):1099-107.

62. Zhao $S, X u$ W, Jiang $W, Y u W$, Lin Y, Zhang $T$, et al. Regulation of cellular metabolism by protein lysine acetylation. Science. 2010;327(5968):1000-4.

63. Winyard PG, Ryan B, Eggleton P, Nissim A, Taylor E, Lo Faro ML, et al. Measurement and meaning of markers of reactive species of oxygen, nitrogen and sulfur in healthy human subjects and patients with inflammatory joint disease. Biochem Soc Trans. 2011;39(5):1226-32.

64. Klareskog L, Catrina Al. Autoimmunity: Lungs and citrullination. Nat Rev Rheumatol. 2015;11(5):261-2.

Ready to submit your research? Choose BMC and benefit from:

- fast, convenient online submission

- thorough peer review by experienced researchers in your field

- rapid publication on acceptance

- support for research data, including large and complex data types

- gold Open Access which fosters wider collaboration and increased citations

- maximum visibility for your research: over $100 \mathrm{M}$ website views per year

At BMC, research is always in progress.

Learn more biomedcentral.com/submissions 